\title{
Sequence Variation in Hepatitis E Virus Genotypes 3 and 4 from Swine Fecal Samples in Japan
}

\author{
Sompong Sapsutthipas ${ }^{1}$, Takeru Urayama ${ }^{1,2}$, Masanobu Yamate ${ }^{1}$, Muneo Tsujikawa ${ }^{2}$, Hiromi Nishigaki ${ }^{2}$, \\ Katsuro Hagiwara ${ }^{3}$, Mikihiro Yunoki ${ }^{1,2}$, Hiroshi Yasue ${ }^{4}$, Kunio Sato and Kazuyoshi Ikuta ${ }^{*}, 1$
}

\author{
${ }^{I}$ Department of Virology, International Center for Infectious Disease Control, Research Institute for Microbial \\ Diseases, Osaka University, Suita, Osaka 565-0871, Japan \\ ${ }^{2}$ Infectious Pathogen Research Group, Osaka Laboratory, Benesis Corporation, Yodogawa-ku, Osaka 532-2488, Japan \\ ${ }^{3}$ Department of Veterinary Microbiology, School of Veterinary Medicine, Rakuno Gakuen University, Ebetsu, Hokkaido \\ 069-8501, Japan
}

${ }^{4}$ Division of Genome and Biodiversity Research, National Institute of Agrobiological Sciences, Tsukuba, Ibaraki 3058602, Japan

${ }^{5}$ Epidemiological Research Team, National Institute of Animal Health, Tsukuba, Ibaraki 305-0856, Japan

\begin{abstract}
Hepatitis E virus (HEV) is a causative agent for hepatitis. HEV is transmitted via the fecal-oral route through contaminated drinking water and induces zoonotic infections through eating uncooked and undercooked meat of deer, wild boar, and swine. In Japan, genotypes $3(\mathrm{G} 3)$ and $4(\mathrm{G} 4)$ are prevalent in domestic swine. Here, we examined the genetic variation among HEVs derived from swine fecal samples in Japan. A total of 320 samples were collected at 32 commercial farm facilities (1 fecal sample from each of 10 pig houses in individual farms). Viral RNA amplification at open reading frame (ORF) 3 was possible in 159 (49.7\%) of the fecal samples. For genotyping, the same samples were subjected to amplification at ORF2 and the resulting amplicons were sequenced. The results revealed that all the HEVs in each farm belonged to the same cluster of G3 and G4: G3 $3_{\mathrm{JP}}$ in 8 farms, G3 $3_{\mathrm{SP}}$ in 4 farms, G3 $3_{\mathrm{US}}$ in 6 farms, and G4 $4_{\mathrm{JP}}$ in 2 farms, unclassified G3 in 2 farms, unable to decide due to a low rate of amplification in 5 farms, and no detection in 5 farms. Interestingly, the HEVs from one farm were more homogeneous than those of the same cluster that was derived from other farms. Thus, the efficiency of farm-to-farm transmission of HEVs is likely to be low and HEV seems to have evolved independently at each farm in Japan.
\end{abstract}

Keywords: HEV, feces, swine, Japan, genotype, food borne, transmission.

\section{INTRODUCTION}

Hepatitis E virus (HEV) is a causative agent for hepatitis [1-3]. Transmission of this virus occurs primarily by the fecal-oral route through contaminated drinking water in areas with poor sanitation [3]. In addition, zoonotic food-borne transmission of HEV from domestic swine, wild boar and wild deer after the ingestion of uncooked or undercooked meat, such as raw liver and colon/intestines, has been reported [4-7]. Although the mortality rate during epidemics is $<1 \%$, it is significantly higher in pregnant women, i.e., $\sim 20 \%[8,9]$

HEV belongs to the genus Hepevirus in the family Hepeviridae [10]. The virion does not possess an envelope and is approximately $27-34 \mathrm{~nm}$ in diameter. Its genome consists of a single-stranded, positive-sense RNA of approximately 7.3 kilobases $(\mathrm{kb})$ and contains a short 5 , untranslated region (5'UTR), three open reading frames (ORF1, ORF2, and ORF3) and a short 3'UTR terminated by

*Address correspondence to this author at the Department of Virology, International Center for Infectious Disease Control, Research Institute for Microbial Diseases, Osaka University, Suita Osaka 565-0871, Japan; Tel.: +8166879 8307; Fax: +8166879 8310; E-mail: ikuta@biken.osaka-u.ac.jp a poly A tract $[3,11]$. HEVs have been classified into genotypes 1-4 (G1-G4) [10, 12]. The majority of HEV genotypes are classified according to geographical prevalence: G1, in Asia and Africa; G2, in Mexico and Africa; G3, in broad areas isolated from sporadic cases of acute hepatitis E and/or domestic swine in USA, European countries, Thailand and Japan; and G4, broadly isolated from humans and/or domestic swine in several Asian countries including China, Taiwan and Japan $[12,13]$.

There have been reports supporting that swine serve as reservoirs for HEV infection in humans [6, 14-18]. Further, significantly higher prevalence of anti-HEV antibody among pig handlers than control subjects was reported [14-16]. In addition, a high genetic relatedness was observed between $\mathrm{HEV}$ isolates from humans and from swine in the same geographical regions [17].

In Japan, a high prevalence of swine anti-HEV antibodies (71\%) among swine of 3-6 months of age and a high viremia rate $(11 \%)$ among swine of $2-4$ months of age have been reported [18, 19]. At least $25(78 \%)$ of 32 patients with sporadic cases of acute hepatitis $E$ in Hokkaido, the northernmost island of Japan, had consumed grilled or undercooked swine liver and/or intestine approximately 2-8 
weeks prior to the onset of hepatitis E [20]. Swine liver specimens from $7(1.9 \%)$ of 363 packages sold in local grocery stores in Hokkaido had detectable HEV RNA, with a viral load of $10^{2}-10^{7}$ copies/g [6]. Importantly, seven swine $\mathrm{HEV}$ isolates recovered from packaged swine livers were found to be very closely related to viruses recovered from patients with hepatitis $\mathrm{E}$ on this island. In addition, there is evidence of people being infected by HEV after ingesting raw meat of infected deer and wild boars [4-6]. Thus, a high prevalence of antibodies to HEV in domestic swine and transmission among swine through a fecal-oral route similar to that in human cases are suspected in Japan [21]. Further, evidence that swine could be a source of transmission to humans leading to acute hepatitis has accumulated through extensive characterization of genetic relatedness, especially in $\mathrm{G} 3$, mostly forming three clusters, $\mathrm{G} 3_{\mathrm{JP}}, \mathrm{G} 3_{\mathrm{SP}}$ and $\mathrm{G} 3_{\mathrm{US}}$, and $\mathrm{G} 4$ forming one cluster, $\mathrm{G} 4_{\mathrm{JP}}[6,18]$. Indigenous acute hepatitis E infections caused by G3 and G4 in Japan were compared for differences in clinical features. It was revealed that patients with genotype 4 had significantly higher peak of aminotransferase levels and also a longer medium duration of hospital stay than those with genotype $3[22,23]$. Genetic variation among HEVs in swine fecal samples is less well characterized, except for $\mathrm{G} 3_{\mathrm{JP}}[6,18,24]$, since most reports have focused on HEVs during viremia in natural infections. However, to understand swine-to-swine and farm-to-farm transmissions of HEV in the field, a more direct approach using a large number of swine fecal samples is needed. In this study, we examined a total of 320 swine fecal samples from 32 farms in Japan to reveal the prevalence of HEV RNA and analyzed their genetic variation. Interestingly, our results revealed that all the HEVs in each farm belonged to the same cluster, $\mathrm{G} 3_{\mathrm{JP}}, \mathrm{G} 3_{\mathrm{SP}}, \mathrm{G} 3_{\mathrm{US}}$, or $\mathrm{G} 4_{\mathrm{JP}}$. In addition, the HEVs from one farm were more homogeneous than those from the other farms belonging to the same cluster.

\section{MATERIALS AND METHODS}

\section{Fecal Samples from Swine in Japan}

A total of 320 fecal samples from swine at 32 commercial pig farms (1 sample from each of 10 pig houses in individual farms) in Japan were obtained for detection of HEV RNA by reverse transcription (RT)-polymerase chain reaction (PCR). The fecal samples were derived from 13 farms in Hokkaido island; 7 farms in the Kanto region of main island; 3 farms in the Chubu and Hokuriku regions of main island; 6 farms in Kyushu and Shikoku islands; and 3 farms in other regions. Permission for sample collection was granted with the condition of not revealing the detailed geographical locations for social reasons. Based on a report of a high prevalence of HEV in swine of 3-4 months of age on farms in Japan [18], we focused on fecal samples from swine in this age group.

Ten fecal samples were carefully obtained using a special tube for the pig fecal sampling. The suckling broods born at the same day in individual pig houses, and not brought from external sources, were reared continuously in the same house, indicating that HEV detected in the suckling pigs was either derived from the mother pigs and/or brothers/sisters. Ten percent (wt/vol) suspensions of about $100 \mathrm{mg}$ of fecal sample in $1 \mathrm{ml}$ of phosphate-buffered saline (PBS; $0.01 \mathrm{M}$, $\mathrm{pH} 7.2-7.4)$ were centrifuged at $10,000 \mathrm{rpm}$ for $10 \mathrm{~min}$ at $4^{\circ} \mathrm{C}$. The supernatant was transferred to a sterilized RNasefree microfuge tube. The clarified $10 \%$ suspensions of fecal samples were stored at $-80^{\circ} \mathrm{C}$ prior to use.

\section{Detection of HEV RNA in Fecal Swine Samples}

Total RNA was extracted from fecal suspensions using a QIAamp Viral RNA Kit (QIAGEN, Hilden, Germany). The copy number of HEV RNA was assessed by quantitative real-time RT-PCR with a primer set (HE86 and HE87) and probe (FHE88) at the ORF3 region, according to Jothikumar et al. [25] with a slight modification (Fig. 1). The primers and probe were synthesized by Applied Biosystems. The 50$\mu 1$ reaction mixture contained $25 \mu 1$ of 2 x QuantiTect Probe RT-PCR kit Master Mix (QIAGEN), $0.5 \mu 1$ of enzyme mixture, $10 \mu \mathrm{l}$ of RNA sample, $13 \mu \mathrm{l}$ of RNase-free distilled water, $400 \mathrm{nM}$ of the primers HE86 and HE87, and $100 \mathrm{nM}$ of the TaqMan ${ }^{\circledR}$ probe FHE88. The samples were run using Applied Biosystems 7500 Realtime PCR system (Applied Biosystems, Foster City, CA).

Reverse transcription was carried out at $50^{\circ} \mathrm{C}$ for $30 \mathrm{~min}$, followed by denaturation at $95^{\circ} \mathrm{C}$ for $15 \mathrm{~min}$. DNA was amplified immediately with 45 cycles at $95^{\circ} \mathrm{C}$ for $15 \mathrm{~s}$ and $60^{\circ} \mathrm{C}$ for $35 \mathrm{~s}$. FHE88 for detecting PCR amplicons was designed based on the sequence of the ORF3 region. An ORF3-containing plasmid was constructed from swine $\mathrm{G} 4_{\mathrm{JP}}$ strain using pCRII-TOPO (Invitrogen) containing T7 promoter. To construct a standard curve for quantification, RNA fragment was transcribed in vitro from the cloned plasmid using T7 polymerase. The copy number of standard RNA was calculated with spectrophotometer.

\section{Genotyping of HEV-Positive Samples}

Total RNA was extracted from fecal suspensions using a QIAamp Viral RNA Kit (QIAGEN). The RNA was reversetranscribed with Prime Script ${ }^{\mathrm{TM}}$ One Step RT-PCR (TAKARA Biomedicals, Shiga, Japan) and subjected to semi-nested PCR in the presence of TaKaRa Ex Taq (TAKARA Biomedicals). Part of the ORF2 sequence was amplified using the primers HE044 and HE040 for the first round of amplification, followed by HE044 and an another primer, HE041, for the second round (Fig. 1). The PCR amplification was carried out for 35 cycles in the same conditions for the first and second rounds $\left[94^{\circ} \mathrm{C}, 30 \mathrm{~s}\right.$ (additional $1 \mathrm{~min}$ in the first cycle); $60^{\circ} \mathrm{C}, 30 \mathrm{~s} ; 72^{\circ} \mathrm{C}, 30 \mathrm{~s}$ (additional $5 \mathrm{~min}$ in the last cycle)]. The size of amplified products was 506 base pairs (bp) for the first-round PCR and $467 \mathrm{bp}$ for the second round PCR. The products were electrophoresed in 1.5\% (wt/vol) Agarose-RE (nacalai tesque, Kyoto, Japan) and stained with ethidium bromide, then photographed under ultraviolet light.

\section{Sequence Analysis of PCR Products at ORF2}

The products were extracted from the agarose gel using a QIAEX ${ }^{\mathrm{R}}$ II Gel Extraction Kit (QIAGEN). Both strands of the products were sequenced using the BigDye Terminator Cycle Sequencing Ready Reaction Kit (Applied Biosystems). Sequence analysis was performed using Genetyx-Mac version 10.1.6 (Software Development, Tokyo, Japan). Sequence alignments were generated by CLUSTAL W (version 1.83) [26]. A phylogenetic tree was constructed by the neighbor-joining method [27], based on the partial nucleotide sequence of the ORF2 region (412 nt). 


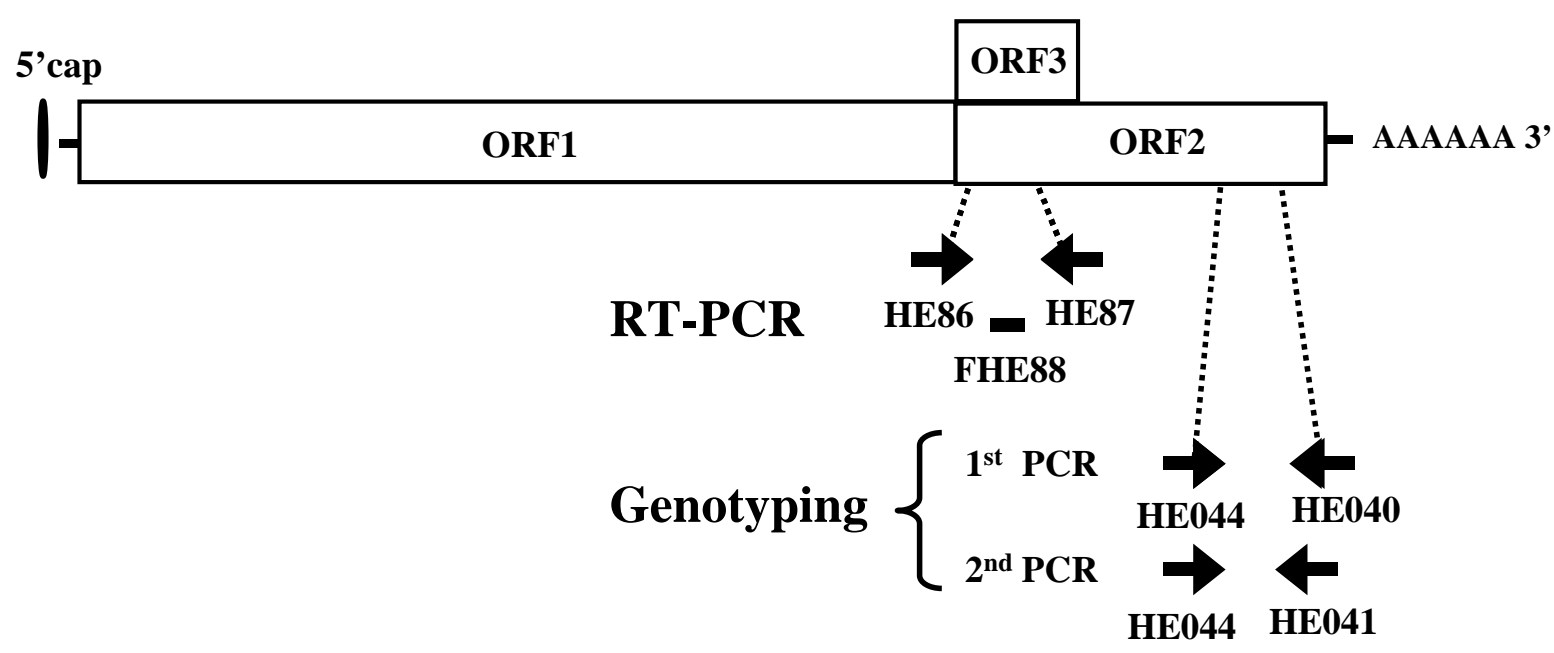

\begin{tabular}{|c|c|}
\hline HE86 (5286 -5303)*; & GGT GGT TTC TGG GGT GAC \\
\hline HE87 (5355 -5338)*; & AGG GGT TGG TTG GAT GAA \\
\hline FHE88 (5309-5326)*; & 5’-FAM-TGA TTC TCA GCC CTT CGC-TAMRA-3’ \\
\hline HE044 (5937 -5959)*; & CAA GGH TGG CGY TCK GTT GAG AC [ $\mathrm{H}=\mathrm{A}, \mathrm{T}$ or $\mathrm{C} ; \mathrm{Y}=\mathrm{T}$ or $\mathrm{C} ; \mathrm{K}=\mathrm{G}$ or $\mathrm{T}$ ] \\
\hline HE040 (6442-6420)*; & CCCTTRTCCTGCTGAGCRTTCTC \\
\hline HE041 (6403-6381)*; & TTM ACW GTC RGC TCG CCA TTG GC [ $\mathrm{M}=\mathrm{A}$ or $\mathrm{C}$; $\mathrm{W}=\mathrm{A}$ or $\mathrm{T} ; \mathrm{R}=\mathrm{A}$ or $\mathrm{G}$ ] \\
\hline
\end{tabular}

*Nucleotide position of swJ570; AB073912

Fig. (1). Location, direction, and sequences of the primers used for RT-PCR. The primers (HE86 and HE87) and probe (FHE88) at the ORF3 region were used to detect HEV RNA in the swine fecal samples. The HEV RNA-positive fecal samples were further subjected to RT-PCR with primers (HE044 and HE040 for the $1^{\text {st }}$ amplification and HE044 and HE041 for the $2^{\text {nd }}$ amplification) at the ORF2 region. The sequences of the primers and probe are shown.

Bootstrap values were determined based on 1000 resamplings of the data sets [28]. The final tree was obtained using the Tree View program (version 1.6.6) [29].

As reference sequences, the following HEVs derived from samples in Japan that were available from GenBank were used: as G3 ${ }_{\text {JP }}$, HE-JA6 (AB082562), wbJYG1 (AB222184), HE-JA21 (AB115542), swJ570 (AB073912), swJ24-1 (AB094306), wbJTS1 (AB222183), JMNG-Oki02C (AB236320), HE-JBD1 (AB112743), JDEER-Hyo3L (AB189071), JRA1 (AP003430), HEVN1 (AB246676), HE-JA23 (AB115544), HE-JA5 (AB082561), swJ681 (AB073910), swJC1990 (AB096756), wbJSG1 (AB222182), HE-JA11 (AB082567), JJT-Kan (AB091394), HE-JA9 (AB082565), swJL97 (AB105899), HEJBD2 (AB154829), HE-JO-1982 (AB088418), HE-JF2 (AB079763), JBOAR1-Hyo04 (AB189070), JMO-Hyo03L (AB189072), JSO-Hyo03L (AB189073), JTH-Hyo03L (AB189074), JYO-Hyo03L (AB189075), HE-JBD3 (AB154830), swJ23-1 (AB094305), swJ19-1 (AB094279), SwJL82 (AB105898), SwJ22-4 (AB094296), SwJ25-1

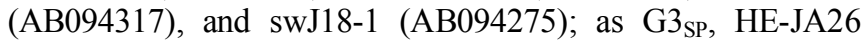
(AB194284), swJ5-1 (AB094216), HEV-Sendai (AB093535), HE-JA04-1911 (AB248520), swJ791 (AB073911), swJ8-5 (AB094230), swJ8-2 (AB094227), swJ12-4 (AB094253), swJ12-1 (AB094250), swJ8-8 (AB094233), and swJ8-6 (AB094231); as G3 ${ }_{\text {us: }}$ HE-JA4 (AB082560), swJ1-1 (AB094203), JKN-Sap (AB074918), JMY-Haw (AB074920), swJ3-1 (AB094212), swJ4-1 (AB094215), swJ11-1 (AB094240), swJ6-1 (AB094217), HE-JA7 (AB082563), HE-
JI3 (AB080579), HE-JA8 (AB082564), HE-JA10 (AB089824), swJL325 (AB105904), swJL234 (AB105903), HE-JA16 (AB105892), swJ2-1 (AB094207), swJ16-1 (AB094267), swJ17-1 (AB094272), swJ10-1 (AB094238), HE-JAS1 (AB107366), HE-JA22 (AB115543), HE-JA20 (AB115541), HE-JAS3 (AB107368), and HE-JA15 (AB105891); and as G4 JP, HE-JA1 (AB097812), swJ13-1 (AB097811), JSN-SapFH02C (AB200239), swJ7-5 (AB094223), HE-JI4 (AB080575), JAK-Sai (AB074915), HE-JA2 (AB220974), HEJA17 (AB105893), HE-JAS2 (AB107367), JKK-Sap (AB074917), swJL145 (AB105902), HE-JA28 (AB220976), HE-JA13 (AB105889), HE-JA14 (AB105890), HE-JA12 (AB105888), HE-JF5 (AB220973), HE-JF4 (AB220972), JTSSap02 (AB161718), JYW-Sap02 (AB161719), HE-JA19 (AB220975), JSM-Sap95 (AB161717), HE-JA18 (AB105894), HE-JA3 (AB082559), HE-JF3 (AB220971), HE-JK4 (AB099347), JSN-Sap-FH (AB091395), and HE-J1 (AB082545).

\section{RESULTS}

\section{Detection of HEV RNA in Swine Fecal Samples in Japan by RT-PCR at ORF3 Region}

A total of 320 fecal samples from 32 (\#1 to \#32) farms were tested for HEV RNA by RT-PCR using ORF3 primers (Fig. 1): ten fecal samples were randomly prepared from pig houses (1 sample/house) on individual farms. The sensitivity to detect HEV RNA was $10^{3.6}$ copies/g feces by real-time RT-PCR using synthetic HEV RNA (data not shown). HEV 
RNA was detected in 159 (49.7\%) of the fecal samples, with extensive variation in the rate of detection among the farms, ranging from $0 \%$ to $100 \%$, as summarized in Table 1 .

\section{Genotyping of HEVs from the Swine Fecal Samples in Japan}

Next, we examined the sequence variation in these samples by RT-PCR using ORF2 primers (Fig. 1). As shown by the phylogenetic tree (Fig. 2A), the HEVs derived from one farm were quite similar and were further well distinguished from those from the other farms. Therefore, one representative sequence from individual farms was randomly selected and used for the next phylogenetic tree, together with already reported HEV sequences from GenBank as references (Fig. 2B). As a consequence, the HEV sequences from 8 (19 samples), 4 (16 samples), 6 (50

Table 1. Summary of HEV RNA in Fecal Samples from Swine in Japan

\begin{tabular}{|c|c|c|c|c|c|c|c|c|}
\hline \multirow{2}{*}{ Farm } & \multirow{2}{*}{$\begin{array}{l}\text { HEV-RNA }{ }^{+} \text {in } 10 \\
\text { Houses }\end{array}$} & \multicolumn{5}{|c|}{ Cluster } & \multirow{2}{*}{$\begin{array}{l}\text { Too Low Amount } \\
\text { for Genotyping* }\end{array}$} & \multirow{2}{*}{ Not Done ${ }^{\dagger}$} \\
\hline & & $\mathbf{G 3}_{\mathrm{JP}}$ & $\mathbf{G} \mathbf{3}_{\mathrm{SP}}$ & $\mathbf{G 3}_{\text {US }}$ & $\mathbf{G 4}_{\mathrm{JP}}$ & $\mathbf{G} 3_{\text {Unclassified }}$ & & \\
\hline \#1 (swJB-A) & $10 / 10$ & 0 & 0 & 10 & 0 & 0 & 0 & 0 \\
\hline \#2 (swJB-B) & $8 / 10$ & 0 & 0 & 8 & 0 & 0 & 0 & 0 \\
\hline \#3 (swJB-C) & $10 / 10$ & 0 & 0 & 10 & 0 & 0 & 0 & 0 \\
\hline \#4 (swJB-D) & $8 / 10$ & 0 & 4 & 0 & 0 & 0 & 4 & 0 \\
\hline \#5 (swJB-E) & $7 / 10$ & 0 & 3 & 0 & 0 & 0 & 3 & 1 \\
\hline \#6 (swJB-F) & $9 / 10$ & 0 & 8 & 0 & 0 & 0 & 1 & 0 \\
\hline \#7 (swJB-G) & $1 / 10$ & 0 & 0 & 0 & 0 & 0 & 1 & 0 \\
\hline \#8 (swJB-H) & $9 / 10$ & 0 & 0 & 0 & 9 & 0 & 0 & 0 \\
\hline$\# 9$ (swJB-I) & $9 / 10$ & 0 & 0 & 9 & 0 & 0 & 0 & 0 \\
\hline \#10 (swJB-J) & $4 / 10$ & 0 & 0 & 0 & 3 & 0 & 1 & 0 \\
\hline \#11 (swJB-K) & $9 / 10$ & 0 & 0 & 9 & 0 & 0 & 0 & 0 \\
\hline \#12 (swJB-L) & $1 / 10$ & 0 & 1 & 0 & 0 & 0 & 0 & 0 \\
\hline \#13 (swJB-M) & $4 / 10$ & 0 & 0 & 4 & 0 & 0 & 0 & 0 \\
\hline \#14 (swJR-A) & $1 / 10$ & 0 & 0 & 0 & 0 & 0 & 1 & 0 \\
\hline \#15 (swJR-B) & $3 / 10$ & 2 & 0 & 0 & 0 & 0 & 1 & 0 \\
\hline \#16 (swJR-C) & $4 / 10$ & 0 & 0 & 0 & 0 & 2 & 0 & 2 \\
\hline \#17 (swJR-D) & $0 / 10$ & 0 & 0 & 0 & 0 & 0 & 0 & 0 \\
\hline \#18 (swJR-E) & $10 / 10$ & 2 & 0 & 0 & 0 & 0 & 0 & 8 \\
\hline \#19 (swJR-F) & $10 / 10$ & 0 & 0 & 0 & 0 & 2 & 0 & 8 \\
\hline \#20 (swJR-G) & $0 / 10$ & 0 & 0 & 0 & 0 & 0 & 0 & 0 \\
\hline \#21 (swJR-H) & $2 / 10$ & 0 & 0 & 0 & 0 & 0 & 2 & 0 \\
\hline \#22 (swJR-I) & $1 / 10$ & 0 & 0 & 0 & 0 & 0 & 1 & 0 \\
\hline \#23 (swJR-J) & $1 / 10$ & 0 & 0 & 0 & 0 & 0 & 1 & 0 \\
\hline \#24 (swJR-K) & $0 / 10$ & 0 & 0 & 0 & 0 & 0 & 0 & 0 \\
\hline \#25 (swJR-L) & $7 / 10$ & 2 & 0 & 0 & 0 & 0 & 0 & 5 \\
\hline \#26 (swJR-M) & $1 / 10$ & 1 & 0 & 0 & 0 & 0 & 0 & 0 \\
\hline \#27 (swJR-N) & $8 / 10$ & 2 & 0 & 0 & 0 & 0 & 0 & 6 \\
\hline \#28 (swJR-O) & $10 / 10$ & 2 & 0 & 0 & 0 & 0 & 0 & 8 \\
\hline \#29 (swJR-P) & $7 / 10$ & 6 & 0 & 0 & 0 & 0 & 1 & 0 \\
\hline \#30 (swJR-Q) & $5 / 10$ & 2 & 0 & 0 & 0 & 0 & 0 & 3 \\
\hline \#31 (swJR-R) & $0 / 10$ & 0 & 0 & 0 & 0 & 0 & 0 & 0 \\
\hline \#32 (swJR-S) & $0 / 10$ & 0 & 0 & 0 & 0 & 0 & 0 & 0 \\
\hline Total & $159 / 320$ & 19 & 16 & 50 & 12 & 4 & 17 & 41 \\
\hline
\end{tabular}

*The amounts amplified by RT-PCR at ORF2 were too low for genotyping.

'Since randomly selected HEV samples showed similar sequences, the remaining samples from the same farm were not subjected to sequencing. 
samples), and 2 (12 samples) farms were classified into G3 $\mathrm{G} 3_{\mathrm{SP}}, \mathrm{G} 3_{\mathrm{US}}$, and $\mathrm{G} 4_{\mathrm{JP}}$, respectively, as summarized in Table 1. The HEVs from farms \#16 (2 samples) and \#19 (2 samples) showed similar sequences to G3, but were located in a different cluster of $\mathrm{G} 33_{\mathrm{JP}}, \mathrm{G} 3_{\mathrm{SP}}$ and $\mathrm{G} 3_{\mathrm{US}}$ (named $\mathrm{G} 3_{\text {Unclassied }}$ in Fig. 2B). A total of 17 samples from farms \#7, $\# 14, \# 21$, \#22 and \#23 showed too weak bands to permit direct sequencing (Table 1). In addition, the amplified RNA amounts of several samples from farms \#4, \#5, \#6, \#10, \#15 and \#29 were also too small for genotyping, but the other samples from these farms could be efficiently amplified for sequencing (Table 1). Thus, the HEVs detected in the samples derived from the same farms were highly homogeneous and therefore, a total of 41 samples from farms $\# 5, \# 16, \# 18, \# 19, \# 25, \# 27, \# 28$ and \#30 were not subjected to sequencing.

\section{Phylogenetic Analysis of Swine-Derived HEVs with Reported Swine- and Human-Derived HEVs}

Next, we characterized the sequence variation in individual clusters of G3 and G4 HEVs in this study by constructing a phylogenetic tree that included reported isolates of HEVs derived from samples in Japan (Fig. 3). One representative sequence per pig farm was used for the tree construction. The data showed that among the sequences belonging to the same clusters of G3, there were considerable variations.
The HEVs belonging to G3 JP were heterogeneous, except for the HEVs that were isolated from the same origin, such as those from deer and humans infected by eating deer meat [5]. All the HEVs that were classified into G3 ${ }_{\mathrm{JP}}$ in this study were remarkably different from the reported isolates of G3 3 derived from humans and swines. Analysis revealed that the swine G3 JP HEVs in this study shared 95.9-86.3\% nucleotide sequence identity to previously reported HEVs of swine, human, boar or deer origin.

In the case of $\mathrm{G} 3_{\mathrm{SP}}$, swJB-E10 and swJB-F1 were far from the reported isolates derived from humans. Most isolates of the HEVs that were classified into $\mathrm{G} 3_{\mathrm{SP}}$ in this study were close to those of reported swine isolates. Further analysis revealed that the swine $\mathrm{G} 3{ }_{\mathrm{SP}} \mathrm{HEVs}$ in this study shared $99.5-89.1 \%$ nucleotide sequence identity to HEVs of swine or human origin.

In the case of $\mathrm{G} 3_{\mathrm{US}}$, most isolates of the HEVs in this study were close to those of reported swine isolates but not human isolates as well as G3 3 sp. swJB-B1 and swJB-M8 were very close, although the HEV sequences were essentially heterogeneous among pig farms in this study. The analysis revealed that the HEVs that were classified into $\mathrm{G}_{\mathrm{US}}$ in this study shared $99.8-89.1 \%$ nucleotide sequence identity to HEVs of swine and human origin.

In the case of $\mathrm{G}_{\mathrm{JP}}$, lot of isolates from humans have been reported and seem to be genetically divided into several
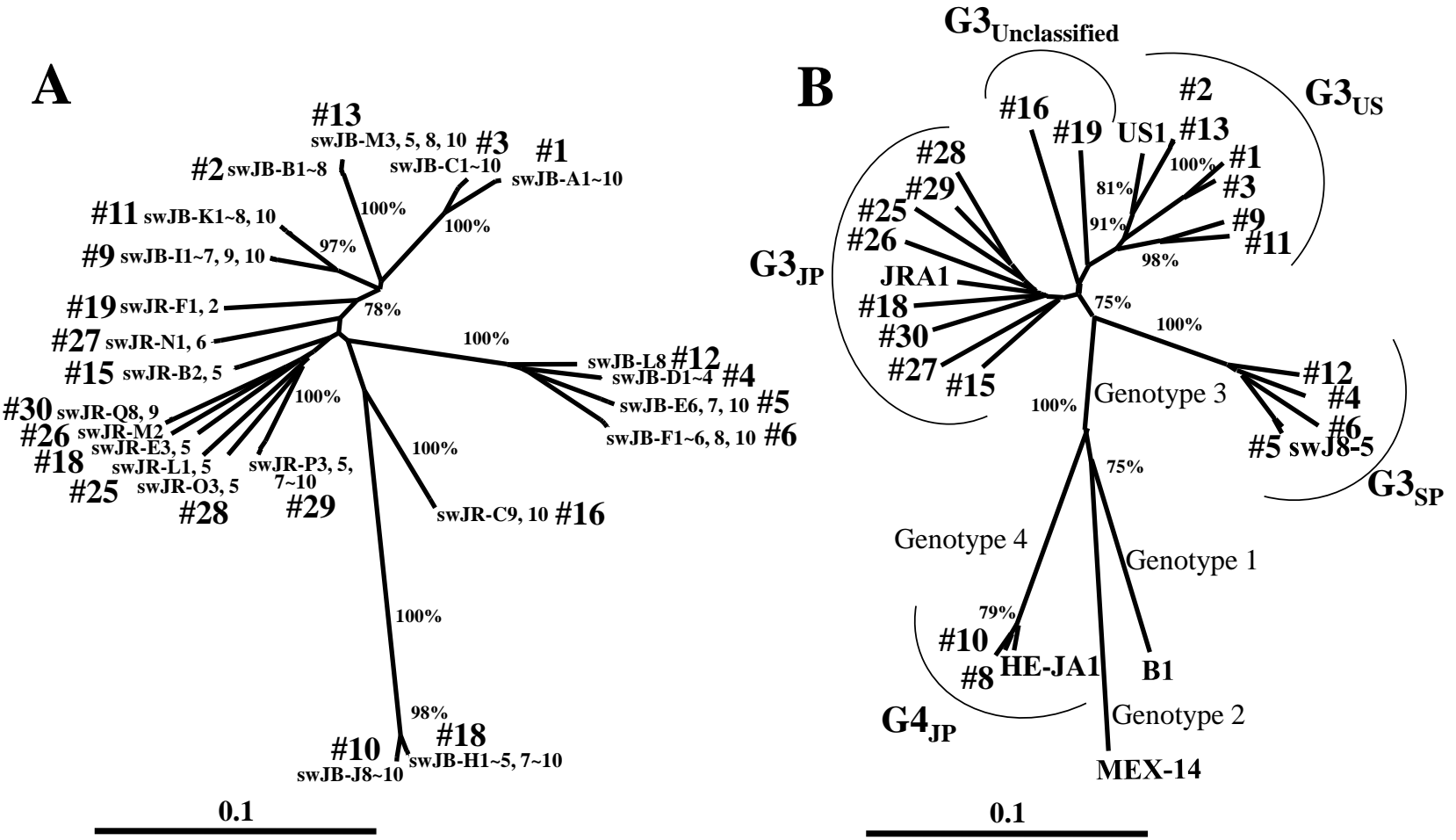

Fig. (2). Phylogenetic tree at ORF2. The phylogenetic tree was constructed based on the partial nucleotide sequence (412 nucleotides) at ORF2 of HEVs of a total of 101 swine fecal samples. A, the $101 \mathrm{HEV}$ samples available for sequencing after RT-PCR with primers at the ORF2 region (Fig. 1) were subjected to phylogenetic analysis. B, HEV representative samples from each farm (\#1, swJB-A1; \#2, swJB-B1; \#3, swJB-C1; \#4, swJB-D1; \#5, swJB-E10; \#6, swJB-F1; \#8, swJB-H7; \#9, swJB-I1; \#10, swJB-J8; \#11, swJB-K1; \#12, swJB-L8; \#13, swJB-M8; \#15, swJR-B2; \#16, swJR-C9; \#18, swJR-E3; \#19, swJR-F1; \#20, ; \#25, swJR-L1; \#26, swJR-M2; \#27, swJR-N1; \#28, swJR-O3; $\# 29$, swJR-P5; and \#30, swJR-Q8) were used for the phylogenetic analysis, together with sequences belonging to genotypes 1 to 4 from the GenBank. Bootstrap values of $>70 \%$ are indicated for the major nodes. The phylogenetic tree was constructed by the neighbor-joining method with 1000 bootstrap value. 


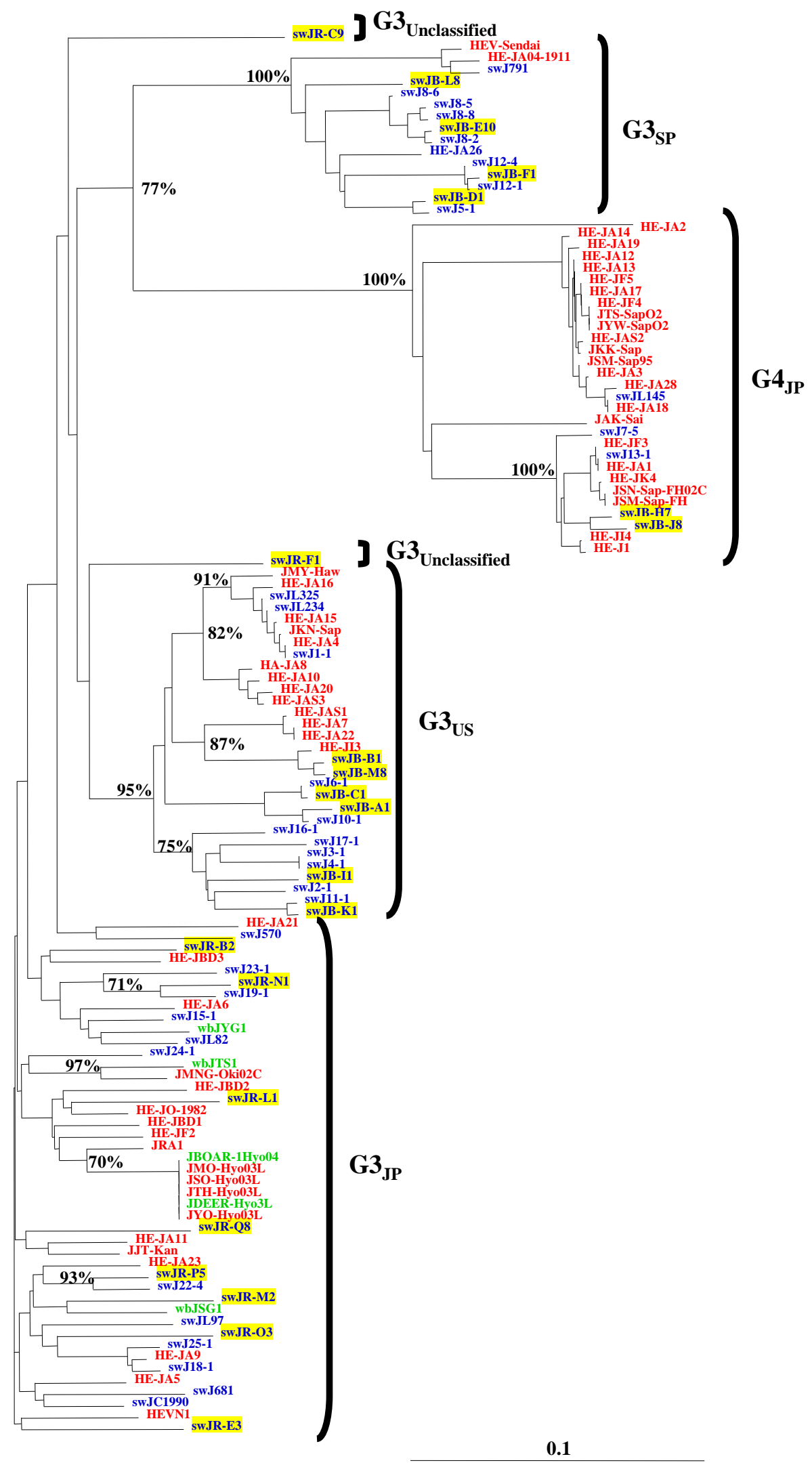

Fig. (3). Phylogenetic analyses of HEV sequences in G3 and G4 clusters. The $101 \mathrm{HEV}$ samples available for sequencing in the ORF2 region were analyzed independently in $\mathrm{G} 3_{\mathrm{JP}}, \mathrm{G} 3_{\mathrm{SP}}, \mathrm{G} 3_{\mathrm{US}}$, and $\mathrm{G} 4_{\mathrm{JP}}$ (highlighted with yellow), together with sequences belonging to individual clusters from the GenBank (red for the HEVs from humans, blue for the HEVs from swine, and green for the HEVs from boar or deer in Japan). Bootstrap values of $>70 \%$ are indicated for the major nodes as a percentage of the data obtained by neighbor-joining method with 1000 boot strap value. 
populations. Thus, although sequence information has been accumulated on human-derived HEVs, there was only limited information on swine-derived G4 $4_{\mathrm{JP}} \mathrm{HEVs}$ in Japan. Both swJB-H7 and swJB-J8 in this study relatively close to several HEVs from humans (Fig. 3). Analysis revealed that the $\mathrm{G}_{\mathrm{JP}} \mathrm{HEVs}$ in this study shared $97.6-87.9 \%$ nucleotide sequence identity to HEVs of swine and human origin.

Similar isolates as G3 $3_{\text {Unclassified }}$ from one (swJR-C9) of the two farms were also previously reported in Japan [19, 30].

\section{DISCUSSION}

The detection rates for HEV RNA in 32 pig farms in Japan were found to be highly variable, ranging from 0 to $100 \%$. In this study, sequence data for the ORF2 region revealed that the HEVs were clearly classified into four known clusters: $\mathrm{G} 3_{\mathrm{JP}}, \mathrm{G} 3_{\mathrm{SP}}, \mathrm{G} 3_{\mathrm{US}}$, and $\mathrm{G} 4_{\mathrm{JP}}$. The HEVs' sequences from one farm showed slight variation even among populations belonging to the same cluster. However, it is worthy to note that the HEVs derived from individual houses within individual pig farms were highly homogeneous. Although some reports showed variation among swine strains circulating in individual farms in Japan $[18,31]$ and Spain [32], others showed similarity of HEVs derived from the same farm [33]. The discrepancy could be due to differences in the size of the farms, distance between farms, degree of contamination with circulating HEVs, and the viral regions sequenced. The results of the present study suggest that farm-to-farm transmission of HEV is very rare; at least between the pig farms examined in this study even though we could not show the detailed geographical location of the sampled farms for permission constraints. Further, the genetic variation found in our swine fecal samples suggests an independent evolution of HEVs in individual pig farm, as previously suggested [34].

The variation in the detection rates of HEV RNA among the pig farms could be caused by several non-viral factors such as sanitary conditions, hosts, facilities, and type of farming, as well as human activities. Although HEV transmission in swine by the fecal-oral route is suspected, it is still unclear how the transmission occurs within or between farms. Further, individually sampled farms reared piglets derived from the same farms, and not from others, suggesting that the identification of closely related sequences belonging to the same cluster in individual farms may be due to long term sustained transmission of HEVs among swine with only minor evolution. In this study, we could not specify the factors responsible for the inter-farm transmission of HEV strains, such as a common animal source, personal relationships, shared water supply, food, or workers.

Further, the HEVs in this study showed phylogenetic similarity to previously reported HEV isolates derived from swine, but only part of them showed similarity to HEVs from humans. Although the relationship between swine- and human-derived HEVs are largely unknown, our data may suggest that swine-derived HEVs could be a possible source for acute hepatitis in humans. Moreover, most of the known human-derived HEVs could have independently evolved after transmission from swine to humans.

In conclusion, swine HEV was highly prevalent in fecal samples from pig farms in Japan. The HEVs in a single farm were genetically highly homogeneous, but showed slightly greater variation to those derived from other farms.

\section{ACKNOWLEDGEMENTS}

We are grateful to Dr. Madiha S. Ibrahim in our institute for valuable discussion and critical reading of the manuscript. This work was conducted based on collaborative research projects between Osaka University and Benesis Corporation and between Rakuno Gakuen University and Benesis Corporation.

\section{REFERENCES}

[1] Reyes GR, Purdy MA, Kim JP, et al. Isolation of a cDNA from the virus responsible for the enterically transmitted non-A, non-B hepatitis. Science 1990; 247: 1335-9.

[2] Arankalle VA, Chadha MS, Tsarev SA, et al. Seroepidemiology of water-borne hepatitis in India and evidence for a third enterically transmitted hepatitis agent. Proc Natl Acad Sci USA 1994; 91: 3428-32.

[3] Purcell RH, Emerson SU. Hepatitis E virus. In: Knipe DM, Howley PM, Griffin DE, Lamb RA, Martin MA, Roizman B, Straus SE, Eds. Fields Virology, $4^{\text {th }}$ ed. Lippincott Williams \& Wilkins, Philadelphia, PA 2001; pp. 3051-61,

[4] Matsuda H, Okada K, Takahashi K, Mishiro S. Severe hepatitis E Virus infection after ingestion of uncooked liver from a wild boar. $\mathrm{J}$ Infect Dis 2003; 188: 944.

[5] Tei S, Kitajima N, Takahashi K, Mishiro S. Zoonotic transmission of hepatitis E virus from deer to human beings. Lancet 2003; 362: 371-3.

[6] Yazaki Y, Mizuo H, Takahashi M, et al. Sporadic acute or fulminant hepatitis E in Hokkaido, Japan, May be food-borne, as suggested by the presence of hepatitis E virus in pig liver as food. J Gen Virol 2003; 84: 2351-7.

[7] Li TC, Chijiwa K, Sera N, et al. Hepatitis E virus transmission from wild boar meat. Emerg Infect Dis 2005; 11: 1958-60.

[8] Hamid SS, Jafri SM, Khan H, et al. Fulminant hepatic failure in pregnant women: acute fatty liver or acute viral hepatitis? J Hepatol 1996; 25: 20-7.

[9] Hussaini SH, Skidmore SJ, Richardson P, et al. Severe hepatitis E infection during pregnancy. J Viral Hepat 1997; 4: 51-4.

[10] Emerson SU, Anderson D, Arankalle A, et al. Hepevirus. In: Fauquet CM, Mayo MA, Maniloff J, Desselberger U, Ball A, Eds. Virus Taxonomy: Eighth Report of the International Committee on Taxonomy of Viruses. London: Elsevier/Academic Press 2004; pp. 851-5.

[11] Wang Y, Zhang H, Ling R, Li H, Harrison TJ. The complete sequence of hepatitis $\mathrm{E}$ virus genotype 4 reveals an alternative strategy for translation of open reading frames 2 and 3. J Gen Virol 2000; 81: 1675-86.

[12] Schlauder GG, Mushahwar IK. Genetic heterogeneity of hepatitis E virus. J Med Virol 2001; 65: 282-92.

[13] Inoue J, Takahashi $\mathrm{M}$, Ito $\mathrm{K}$, Shimosegawa $\mathrm{T}$, Okamoto $\mathrm{H}$ Analysis of human and swine hepatitis E virus (HEV) isolates of genotype 3 in Japan that are only $81-83 \%$ similar to reported HEV isolates of the same genotype over the entire genome. J Gen Virol 2006; 87: 2363-9.

[14] Hsieh SY, Meng XJ, Wu YH, et al. Identity of a novel swine hepatitis $\mathrm{E}$ virus in Taiwan forming a monophyletic group with Taiwan isolates of human hepatitis E virus. J Clin Microbiol 1999; 37: 3828-34.

[15] Drobeniuc J, Favorov MO, Shapiro CN, et al. Hepatitis E virus antibody prevalence among persons who work with swine. J Infect Dis 2001; 184: 1594-7.

[16] Meng XJ, Wiseman B, Elvinger F, et al. Prevalence of antibodies to hepatitis $\mathrm{E}$ virus in veterinarians working with swine and in normal blood donors in the United States and other countries. J Clin Microbiol 2002; 40: 117-22.

[17] Nishizawa T, Takahashi M, Mizuo H, et al. Characterization of Japanese swine and human hepatitis E virus isolates of genotype IV with $99 \%$ identity over the entire genome. J Gen Virol 2003; 84: 1245-51.

[18] Takahashi M, Nishizawa T, Miyajima H, et al. Swine hepatitis E virus strains in Japan form four Phylogenetic clusters comparable 
with those of Japanese isolates of human hepatitis E virus. J Gen Virol 2003; 84: 851-62.

[19] Takahashi M, Nishizawa T, Tanaka T, et al. Correlation between positivity for immunoglobulin A antibodies and viremia of swine hepatitis E virus observed among farm pigs in Japan. J Gen Virol 2005; 86: 1807-13.

[20] Mizuo H, Yazaki Y, Sugawara K, et al. Possible risk factors for the transmission of hepatitis $\mathrm{E}$ virus and for the severe form of hepatitis E acquired locally in Hokkaido, Japan. J Med Virol 2005; 76:341-9.

[21] Okamoto H, Takahashi M, Nishizawa T. Features of hepatitis E virus infection in Japan. Intern Med 2003; 42: 1065-71.

[22] Ohnishi S, Kang JH, Maekubo H, et al. Comparison of clinical features of acute hepatitis caused by hepatitis E virus (HEV) genotypes 3 and 4 in Sapporo, Japan. Hepatol Res 2006; 36: 301-7.

[23] Okamoto H. Genetic variability and evolution of hepatitis E virus. Virus Res 2007; 127: 216-28.

[24] Okamoto H, Takahashi M, Nishizawa T, et al. Analysis of the complete genome indigenous swine hepatitis $\mathrm{E}$ virus isolated in Japan. Biochem Biophys Res Commun 2001; 289: 929-36.

[25] Jothikumar N, Cromeans TL, Robertson BH, Meng XJ, Hill VR. A broadly reactive one-step real-time RT-PCR assay for rapid and sensitive detection of hepatitis E virus. J Virol Methods 2006; 131: 65-71.

[26] Thompson JD, Higgins DG, Gibson TJ. CLUSTAL W: improving the sensitivity of progressive multiple sequence alignment through sequence weighting, position-specific gap penalties and weight matrix choice. Nucleic Acids Res 1994; 22: 4673-80.

[27] Saitou N, Nei M. The neighbor-joining method: a new method for reconstructing phylogenetic tree. Mol Biol Evol 1987; 4: 406-25.

[28] Felsenstein J. Confidence limits on phylogenies: an approach using the bootstrap. Evolution 1985; 39: 783-91.

[29] Page RDM. TREEVIEW: An application to display phylogenetic trees on personal computers. Comput Appl Biosci 1996; 12: 357-8.

[30] Gotanda $\mathrm{Y}$, Iwata $\mathrm{A}$, Ohnuma $\mathrm{H}$, et al. Ongoing subclinical infection of hepatitis E virus among blood donors with an elevated alanine aminotransferase level in Japan. J Med Virol 2007; 79: 734-42.

[31] Nakai I, Kato K, Miyazaki A, et al. Different fecal shedding patterns of two common strains of hepatitis $\mathrm{E}$ virus at three Japanese swine farms. Am J Trop Med Hyg 2006; 75: 1171-77.

[32] De Deus N, Casas M, Peralta B, et al. Hepatitis E virus infection dynamics and organic distribution in naturally infected pigs in farrow-to-finish farm. Vet Microbiol 2008; 132: 19-28.

[33] Ning H, Yu S, Zhu Y, et al. Genotype 3 hepatitis $E$ has been widespread in pig farms of Shanghai suburbs. Vet Microbiol 2008; 126: 257-63.

[34] Di Bartolo I, Martelli F, Inglese N, et al. Widespread diffusion of genotype 3 hepatitis E virus among farming swine in Northern Italy. Vet Microbiol 2008; 132: 47-55.

(C) Sapsutthipas et al.; Licensee Bentham Open.

This is an open access article licensed under the terms of the Creative Commons Attribution Non-Commercial License (http: //creativecommons.org/licenses/by$\mathrm{nc} / 3.0 /$ ) which permits unrestricted, non-commercial use, distribution and reproduction in any medium, provided the work is properly cited. 